\title{
JOINT BANDWIDTH AND POWER ALLOCATION IN COGNITIVE RADIO NETWORKS UNDER FADING CHANNELS
}

\author{
Xiaowen Gong, Sergiy A. Vorobyov, and Chintha Tellambura \\ Department of Electrical and Computer Engineering, University of Alberta, Edmonton, AB, Canada \\ Emails: \{xgong2, vorobyov, chintha\}@ece.ualberta.ca
}

\begin{abstract}
A problem of joint optimal bandwidth and power allocation in cognitive networks under fading channels is considered. It is assumed that multiple secondary users (SUs) share the spectrum of a primary user (PU) using frequency division multiple access. The bandwidth and power are allocated so as to maximize the sum ergodic capacity of all SUs under the total bandwidth constraint of the licensed spectrum as well as different combinations of the peak/average transmit power constraints at the SUs and the peak/average interference power constraint imposed by the PU. Although the optimization problem is convex, its dimension and, thus, complexity may be high. Therefore, computationally efficient ways of solving the problem are of importance and are investigated here by finding structures of the optimal solutions to the problem under different combinations of the constraints.
\end{abstract}

Index Terms - Joint bandwidth and power allocation, cognitive radio, fading channels, sum ergodic capacity

\section{INTRODUCTION}

Spectrum underlay in cognitive radio enables the primary and secondary users (PUs) and (SUs) to transmit simultaneously, provided that the interference power received by the PU is below a prescribed threshold level [1], [2]. Under the setup of a single SU, a number of recent works have studied the information theoretic limits for resource allocation in the context of spectrum underlay (see, for example, [3]). Moreover, the recent work [4] studies a cognitive radio network of multiple SUs under multiple access channel and broadcast channel models. The optimal power allocation maximizing the sum ergodic capacity of the SUs subject to various mixed transmit and interference power constraints has been derived.

In this paper, we extend the results of [4] by considering joint bandwidth and power allocation in cognitive radio networks under a variety of different practical constraints such as the transmit power constraints at the SUs and the interference power constraint imposed by the PU as well as the total bandwidth constraint of the shared spectrum. Moreover, different

This work was supported in parts by the Natural Science and Engineering Research Council (NSERC) of Canada and the Alberta Innovates - Technology Futures, Alberta, Canada. from the existing works, all combinations of the transmit and interference power constraints are considered here.

\section{SYSTEM MODEL}

Consider a cognitive radio network consisting of $N$ SUs and one PU. The PU occupies a spectrum of bandwidth $W$, while the same spectrum is shared by the SUs. The spectrum is divided into distinct and nonoverlapping flat fading channels with different bandwidth, so that the SUs share the spectrum through frequency division multiple access (FDMA) without interfering each other. Denote the instantaneous channel gains between the $i$ th SU transmitter (SU-Tx) and the $i$ th SU receiver (SU-Rx) and between the $i$ th SU-Tx and the PU receiver (PU-Rx) by $h_{i}$ and $g_{i}$, respectively. Then the corresponding instantaneous channel gain vectors are $g \triangleq$ $\left[\begin{array}{llll}g_{1} & g_{2} & \cdots & g_{N}\end{array}\right]$ and $\boldsymbol{h} \triangleq\left[\begin{array}{llll}h_{1} & h_{2} & \cdots & h_{N}\end{array}\right]$, and they represent particular realizations of the channel fading vector random processes $\boldsymbol{G}$ and $\boldsymbol{H}$, respectively, which are assumed to be ergodic and stationary. The statistics of the channel gains ${ }^{1}$ and the instantaneous values of the gains are assumed to be known at the SUs. ${ }^{2}$ The noise at each SU-Rx plus the interference from the PU transmitter (PU-Tx) is assumed to be additive white Gaussian with unit power spectral density (PSD).

Let $p_{i}(\boldsymbol{h}, \boldsymbol{g})$ and $w_{i}(\boldsymbol{h}, \boldsymbol{g})$ denote the transmit power and channel bandwidth allocated to the $i$ th SU-Tx, respectively, at a particular channel fading state $(\boldsymbol{h}, \boldsymbol{g})$. Then the total bandwidth constraint can be expressed as $\sum_{i=1}^{N} w_{i}(\boldsymbol{h}, \boldsymbol{g}) \leq$ $W, \forall \boldsymbol{h}, \boldsymbol{g}$. The peak transmit power (PTP) constraints are given by $p_{i}(\boldsymbol{h}, \boldsymbol{g}) \leq P_{i}^{p k}, \forall i, \boldsymbol{h}, \boldsymbol{g}$ where $P_{i}^{p k}$ denotes the maximum peak transmit power of the $i$ th SUTx. The peak interference power (PIP) constraint is given by $\sum_{i=1}^{N} g_{i} p_{i}(\boldsymbol{h}, \boldsymbol{g}) \leq Q^{p k}, \forall \boldsymbol{h}, \boldsymbol{g}$ where $Q^{p k}$ denotes the maximum peak interference power allowed at the PURx. The average transmit power (ATP) constraints are given by $\mathrm{E}_{\boldsymbol{H}, \boldsymbol{G}}\left\{p_{i}(\boldsymbol{H}, \boldsymbol{G})\right\} \leq P_{i}^{a v}, \forall i$ where the expectation is taken over $\boldsymbol{H}$ and $\boldsymbol{G}$ and $P_{i}^{a v}$ denotes the maximum average transmit power of the $i$ th SU-Tx. The average interference power (AIP) constraint is given by

\footnotetext{
${ }^{1}$ In general, channel statistics are different across different links.

${ }^{2}$ Full CSI assumption is typical in the context of investigating information theoretic limits for cognitive radio [2-4]. The CSI from SU-Tx to PU-Rx can be obtained by methods given in, e.g., [2] and references therein.
} 
$\mathrm{E}_{\boldsymbol{H}, \boldsymbol{G}}\left\{\sum_{i=1}^{N} g_{i} p_{i}(\boldsymbol{H}, \boldsymbol{G})\right\} \leq Q^{a v}$ where $Q^{a v}$ denotes the maximum average interference power allowed at the PU-Rx.

Ergodic capacity [5], which is defined as the maximum average achievable rate, serves as a relevant performance metric for delay-tolerant applications. Thus, our objective is to maximize the sum ergodic capacity of SUs, that is,

$\max _{\left\{w_{i}(\boldsymbol{h}, \boldsymbol{g}), p_{i}(\boldsymbol{h}, \boldsymbol{g}), \forall \boldsymbol{h}, \boldsymbol{g}\right\} \in \mathcal{F}} \mathrm{E}_{\boldsymbol{H}}, \boldsymbol{G}\left\{\left\{\sum_{i=1}^{N} G_{i}\left(w_{i}(\boldsymbol{H}, \boldsymbol{G}), p_{i}(\boldsymbol{H}, \boldsymbol{G})\right)\right\}\right.$

where $G_{i}\left(w_{i}(\boldsymbol{H}, \boldsymbol{G}), p_{i}(\boldsymbol{H}, \boldsymbol{G})\right)$ can be expressed for particular realizations $\boldsymbol{h}$ and $\boldsymbol{g}$ as $G_{i}\left(w_{i}(\boldsymbol{h}, \boldsymbol{g}), p_{i}(\boldsymbol{h}, \boldsymbol{g})\right) \triangleq$ $w_{i}(\boldsymbol{h}, \boldsymbol{g}) \log \left(1+h_{i} p_{i}(\boldsymbol{h}, \boldsymbol{g}) / w_{i}(\boldsymbol{h}, \boldsymbol{g})\right)$ and $\mathcal{F}$ is a feasible set specified by the bandwidth constraints as well as a particular combination of the transmit power constraints PTP, ATP and the interference power constraints PIP, AIP. The constraints on nonnegativity of the bandwidth and power allocations also apply, but are omitted hereafter for brevity. The objective function (1) is concave with respect to $\left\{w_{i}(\boldsymbol{h}, \boldsymbol{g}), p_{i}(\boldsymbol{h}, \boldsymbol{g})\right\}$, $\forall i, \boldsymbol{h}, \boldsymbol{g}$ as well as the bandwidth and power constraints are linear and, thus, convex. Hence, the sum ergodic capacity maximization problem under different combinations of the aforementioned constraints is a convex optimization problem. Our objective is to analyze and find insights to this optimization problem under different combinations of the constraints, which enable us to design computationally efficient algorithms for joint bandwidth and power allocation.

\section{MAIN RESULTS}

A. Optimal Bandwidth Allocation: For fixed power allocation $p_{i}(\boldsymbol{h}, \boldsymbol{g}), \forall i, \boldsymbol{h}, \boldsymbol{g}$, the optimization problem (1) can be represented as maximization of $\mathrm{E}_{\boldsymbol{H}, \boldsymbol{G}}\left\{f_{0}(\boldsymbol{H}, \boldsymbol{G})\right\}$ with $f_{0}(\boldsymbol{h}, \boldsymbol{g})$ for particular realizations $h$ and $g$ given by

$f_{0}(\boldsymbol{h}, \boldsymbol{g}) \triangleq \max _{\left\{w_{i}(\boldsymbol{h}, \boldsymbol{g})\right\}} \sum_{i=1}^{N} G_{i}\left(w_{i}(\boldsymbol{h}, \boldsymbol{g})\right)$ s.t. $\sum_{i=1}^{N} w_{i}(\boldsymbol{h}, \boldsymbol{g}) \leq W$.

This problem is similar to the classical water-filling power allocation problem and its optimal solution, denoted by $\left\{w_{i}^{\prime}(\boldsymbol{h}, \boldsymbol{g})\right\}$, is $w_{i}^{\prime}(\boldsymbol{h}, \boldsymbol{g})=W h_{i} p_{i}(\boldsymbol{h}, \boldsymbol{g}) / \sum_{i=1}^{N} h_{i} p_{i}(\boldsymbol{h}, \boldsymbol{g})$ (see [7]). Substituting this solution into (1), we obtain

$$
\max _{\left\{p_{i}(\boldsymbol{h}, \boldsymbol{g}), \forall \boldsymbol{h}, \boldsymbol{g}\right\} \in \mathcal{F}^{\prime}} \mathrm{E}_{\boldsymbol{H}, \boldsymbol{G}}\left\{H\left(\left\{p_{i}(\boldsymbol{H}, \boldsymbol{G})\right\}\right)\right\}
$$

where $H\left(\left\{p_{i}(\boldsymbol{H}, \boldsymbol{G})\right\}\right) \triangleq W \log \left(1+\sum_{i=1}^{N} h_{i} p_{i}(\boldsymbol{H}, \boldsymbol{G}) / W\right)$ and $\mathcal{F}^{\prime}$ is a feasible set specified by a particular combination of the power constraints. This result enables us to decouple the initially joint problem of bandwidth and power allocation. Indeed, the optimal power allocation $\left\{p_{i}^{*}(\boldsymbol{h}, \boldsymbol{g})\right\}$ can be found first using (2). Then the optimal bandwidth allocation is $w_{i}^{*}(\boldsymbol{h}, \boldsymbol{g})=W h_{i} p_{i}^{*}(\boldsymbol{h}, \boldsymbol{g}) / \sum_{i=1}^{N} h_{i} p_{i}^{*}(\boldsymbol{h}, \boldsymbol{g})$.

B. Optimal Power Allocation under PTP and PIP Constraints: In this case, $\mathcal{F}^{\prime}=\{\mathrm{PTP}, \mathrm{PIP}\}$ and the optimal value of the problem (2) is represented as the maximum of $\mathrm{E}_{\boldsymbol{H}, \boldsymbol{G}}\left\{f_{1}(\boldsymbol{H}, \boldsymbol{G})\right\}$ with $f_{1}(\boldsymbol{h}, \boldsymbol{g})$ for particular realizations $\boldsymbol{h}$ and $\boldsymbol{g}$ given by

$$
\begin{array}{r}
f_{1}(\boldsymbol{h}, \boldsymbol{g}) \triangleq \max _{\left\{p_{i}(\boldsymbol{h}, \boldsymbol{g})\right\}} H\left(\left\{p_{i}(\boldsymbol{h}, \boldsymbol{g})\right\}\right) \\
\text { s.t. } p_{i}(\boldsymbol{h}, \boldsymbol{g}) \leq P_{i}^{p k}, \forall i \\
\sum_{i=1}^{N} g_{i} p_{i}(\boldsymbol{h}, \boldsymbol{g}) \leq Q^{p k} .
\end{array}
$$

Let us omit the dependence on $\boldsymbol{h}$ and $\boldsymbol{g}$ for brevity and denote the optimal solution of the problem (3a)-(3c) by $\left\{p_{i}^{*}\right\}$ as well as denote by $\left(s_{1}, s_{2}, \cdots, s_{N}\right)$ a permutation of the SU indexes such that $h_{s_{1}} / g_{s_{1}}>h_{s_{2}} / g_{s_{2}}>\cdots>h_{s_{N}} / g_{s_{N}}$. It is assumed that $h_{i} / g_{i} \neq h_{j} / g_{j}, \forall i \neq j$, since $h_{i}, g_{i}, h_{j}$, and $g_{j}$ are drawn from a continuous-valued random process. Then the following lemma is constructive for designing a computationally efficient algorithm for solving the joint bandwidth and power allocation problem. ${ }^{3}$

Lemma 1: There exists $k, 1 \leq k \leq N$, such that $p_{s_{i}}^{*}=$ $P_{s_{i}}^{p k}, \forall i, 1 \leq i \leq k-1,0<p_{s_{k}}^{*} \leq P_{s_{k}}^{p k}$, and $p_{s_{i}}^{*}=0, \forall i$, $k+1 \leq i \leq N$.

This lemma stands that for the optimal power allocation under the PTP and PIP constraints, there exists at most one user that transmits at nonzero power and below its peak power, while any other user either does not transmit or transmits at its peak power. This gives the structure of the optimal solution for power allocation.

C. Optimal Power Allocation under ATP and AIP Constraints: In this case, $\mathcal{F}^{\prime}=\{\mathrm{ATP}, \mathrm{AIP}\}$ and the dual function of the problem (2) can be written as $f_{2}\left(\left\{\lambda_{i}\right\}, \mu\right) \triangleq$ $\mathrm{E}_{\boldsymbol{H}, \boldsymbol{G}}\left\{f_{2}^{\prime}(\boldsymbol{H}, \boldsymbol{G})\right\}+\sum_{i=1}^{N} \lambda_{i} P_{i}^{a v}+\mu Q^{a v}$ where $\left\{\lambda_{i} \mid 1 \leq\right.$ $i \leq N\}$ and $\mu$ are the nonnegative dual variables associated with the ATP and AIP constraints, respectively. Moreover, $f_{2}^{\prime}(\boldsymbol{h}, \boldsymbol{g})$ for particular realizations $\boldsymbol{h}$ and $\boldsymbol{g}$ is given by $f_{2}^{\prime}(\boldsymbol{h}, \boldsymbol{g}) \triangleq \max _{\left\{p_{i}(\boldsymbol{h}, \boldsymbol{g})\right\}} H\left(\left\{p_{i}(\boldsymbol{h}, \boldsymbol{g})\right\}\right)-\sum_{i=1}^{N} \gamma_{i} p_{i}(\boldsymbol{h}, \boldsymbol{g})$ with $\gamma_{i} \triangleq \lambda_{i}+\mu g_{i}$. Denote by $\left\{p_{i}^{*}\right\}$ the corresponding optimal power allocation. Then the following lemmas are constructive for building a computationally efficient algorithm.

Lemma 2: If $h_{i} \leq \gamma_{i}$ for some $i$, then $p_{i}^{*}=0$.

Lemma 3: $p_{i}^{*}=0, \forall i$, if and only if $h_{i} \leq \gamma_{i}, \forall i$.

Lemma 4: Let $\left(s_{1}, s_{2}, \cdots, s_{N}\right)$ denote a permutation of the SU indexes such that $h_{s_{1}} / \gamma_{s_{1}}>h_{s_{2}} / \gamma_{s_{2}}>\cdots>$ $h_{s_{N}} / \gamma_{s_{N}}$. Then there exists at most one $k$ such that $p_{k}^{*}>0$. Moreover, $k=s_{1}$.

Thus, for the optimal power allocation under the ATP and AIP constraints, there exists at most one user that transmits at nonzero power, while any other user does not transmit. The following two cases are then of interest: (i) when $h_{i} \leq \gamma_{i}, \forall i$, it follows from Lemma 3 that $p_{i}^{*}=0, \forall i$; (ii) when $h_{i} \leq \gamma_{i}$ does not hold for some $i,\left\{p_{i}^{*}\right\}$ can be found using Lemma 4.

\footnotetext{
${ }^{3}$ For details, we refer the reader to [7].
} 
D. Optimal Power Allocation under PTP and AIP Constraints: In this case, $\mathcal{F}^{\prime}=\{\mathrm{PTP}, \mathrm{AIP}\}$ and the dual function of the problem (2) is $f_{3}(\mu) \triangleq \mathrm{E}_{\boldsymbol{H}, \boldsymbol{G}}\left\{f_{3}^{\prime}(\boldsymbol{H}, \boldsymbol{G})\right\}+\mu Q^{a v}$ where $\mu$ is the nonnegative dual variable associated with the AIP constraint. Here $f_{3}^{\prime}(\boldsymbol{h}, \boldsymbol{g})$ is given by

$$
\begin{gathered}
f_{3}^{\prime}(\boldsymbol{h}, \boldsymbol{g}) \triangleq \max _{\left\{p_{i}(\boldsymbol{h}, \boldsymbol{g})\right\}} H\left(\left\{p_{i}(\boldsymbol{h}, \boldsymbol{g})\right\}\right)-\mu \sum_{i=1}^{N} g_{i} p_{i}(\boldsymbol{h}, \boldsymbol{g}) \\
\text { s.t. } p_{i}(\boldsymbol{h}, \boldsymbol{g}) \leq P_{i}^{p k}, \quad \forall i .
\end{gathered}
$$

Denoting the optimal solution of the problem (4a)-(4b) as $\left\{p_{i}^{*}\right\}$, we consider the following two cases: (i) when $h_{i} \leq$ $\mu g_{i}, \forall i$, it follows from Lemma 3 that $p_{i}^{*}=0, \forall i$; (ii) when $h_{i} \leq \mu g_{i}$ does not hold for some $i$, the following lemma can be stated.

Lemma 5: Let $\left(s_{1}, s_{2}, \cdots, s_{N}\right)$ denote a permutation of the $S U$ indexes such that $h_{s_{1}} / \mu g_{s_{1}}>h_{s_{2}} / \mu g_{s_{2}}>\cdots>$ $h_{s_{N}} / \mu g_{s_{N}}$. Then there exists $k, 1 \leq k \leq N$, such that $p_{s_{i}}^{*}=$ $P_{s_{i}}^{p k}, \forall i, 1 \leq i \leq k-1,0<p_{s_{k}}^{*} \leq P_{s_{k}}^{p k}$, and $p_{s_{i}}^{*}=0, \forall i$, $k+1 \leq i \leq N$.

Lemma 5 is similar to Lemma 1 since it shows that for the optimal power allocation under the PTP and AIP constraints, there exists at most one user that transmits at nonzero power and below its peak power, while any other user either does not transmit or transmits at its peak power.

E. Optimal Power Allocation under ATP and PIP Constraints: In this case, $\mathcal{F}^{\prime}=\{\mathrm{ATP}, \mathrm{PIP}\}$ and the dual function of the problem (2) can be written as $f_{4}\left(\left\{\lambda_{i}\right\}\right) \triangleq$ $\mathrm{E}_{\boldsymbol{H}, \boldsymbol{G}}\left\{f_{4}^{\prime}(\boldsymbol{H}, \boldsymbol{G})\right\}+\sum_{i=1}^{N} \lambda_{i} P_{i}^{a v}$ where $\left\{\lambda_{i} \mid 1 \leq i \leq N\right\}$ are the nonnegative dual variables associated with the ATP constraints. Here $f_{4}^{\prime}(\boldsymbol{h}, \boldsymbol{g})$ is given by

$$
\begin{gathered}
f_{4}^{\prime}(\boldsymbol{h}, \boldsymbol{g}) \triangleq \max _{\left\{p_{i}(\boldsymbol{h}, \boldsymbol{g})\right\}} H\left(\left\{p_{i}(\boldsymbol{h}, \boldsymbol{g})\right\}\right)-\sum_{i=1}^{N} \lambda_{i} p_{i}(\boldsymbol{h}, \boldsymbol{g}) \\
\text { s.t. } \sum_{i=1}^{N} g_{i} p_{i}(\boldsymbol{h}, \boldsymbol{g}) \leq Q^{p k} .
\end{gathered}
$$

Denoting the optimal solution of the problem (5a)-(5b) as $\left\{p_{i}^{*}\right\}$, we consider the following three cases: (i) when $h_{i} \leq \lambda_{i}, \forall i$, it follows from Lemma 3 that $p_{i}^{*}=0, \forall i$; (ii) when $h_{i} \leq \lambda_{i}$ does not hold for some $i$ and the constraint (5b) is inactive at optimality, the optimal solution is similar to that in the case of PTP and AIP constraints, where $\left(s_{1}, s_{2}, \cdots, s_{N}\right)$ is a permutation of the $\mathrm{SU}$ indexes such that $h_{s_{1}} / \lambda_{s_{1}}>h_{s_{2}} / \lambda_{s_{2}}>\cdots>h_{s_{N}} / \lambda_{s_{N}}$; (iii) when $h_{i} \leq \lambda_{i}$ does not hold for some $i$ and the constraint (5b) is active at optimality, the following lemma holds.

Lemma 6: There exists at most two $j \neq k$ such that $p_{j}^{*}>$ 0 and $p_{k}^{*}>0$.

Thus, for the optimal power allocation under the ATP and PIP constraints, there exists at most two users that transmit at nonzero power, while any other user does not transmit.

E. Optimal Power Allocation under a Combination of More Then Two Constraints: It can be shown that under the
PTP, ATP, and AIP constraints and the PIP, ATP, and AIP constraints, the corresponding dual functions of the problem (2) have the same forms as the dual functions in the cases of the PTP+AIP and the ATP+PIP constraints, correspondingly. Moreover, under the PTP, PIP, and ATP constraints and the PTP, PIP, and AIP constraints, the corresponding dual functions have the same form as the dual function in the case of all the PTP, PIP, ATP, and AIP constraints (see [7]). In the latter case, the main result is that for the optimal power allocation, there exists at most two users that transmit at nonzero power and below their peak power, while any other user either does not transmit or transmits at its peak power.

\section{SIMULATION RESULTS}

Consider a cognitive radio network which consists of one PU and four SUs. Rayleigh fading is present in all links and the variance of the channel gain is set to $\sigma^{2}=1$. Moreover, $W=1, P_{i}^{p k}=10, \forall i, P_{i}^{a v}=10, \forall i, Q^{p k}=1$, and $Q^{a v}=1$ are set as default values when fixed. The additive white Gaussian noise with unit PSD is assumed and 1000 randomly generated channel gains for $\boldsymbol{h}$ and $\boldsymbol{g}$ are used. The results are compared under the following five combinations of the power constraints: the PTP+PIP, the PTP+AIP, the ATP+PIP, the $\mathrm{ATP}+\mathrm{AIP}$, and the PTP+ATP+PIP+AIP. The detailed algorithms for all the five cases of different combinations of the constraints can be found in [7].

The maximum sum ergodic capacity under different combinations of power constraints is shown in Figs. 1, 2, 3, and 4 versus $P^{p k}\left(P^{p k}=P_{i}^{p k}, \forall i\right), P^{a v}\left(P^{a v}=P_{i}^{a v}, \forall i\right), Q^{p k}$, and $Q^{a v}$, respectively. It can be seen from Fig. 1 that the capacity achieved under the PTP+AIP constraints is larger than that achieved under the PTP+PIP constraints for any given $P^{p k}$. It is because the AIP constraint allows for more flexibility for SUs to allocate transmit power over different channel fading states than the PIP constraint. Moreover, the performance under the $\mathrm{PTP}+\mathrm{ATP}+\mathrm{PIP}+\mathrm{AIP}$ constraints is close to that under the PTP+PIP constraints because the PTP constraint dominates over the ATP, PIP, and AIP constraints. Fig. 2 shows that the capacity achieved under the ATP+AIP constraints is larger than that achieved under the ATP+PIP constraints because the PIP constraint is stricter than the AIP one. Moreover, the capacity under the PTP+ATP+PIP+AIP constraints is much smaller than that under the ATP+PIP and the ATP+AIP constraints because the PTP constraint is dominant over others. Fig. 3 depicts that the capacity achieved under the ATP+PIP constraints is larger than that achieved under the PTP+PIP constraints for any given $Q^{p k}$ because the power allocation is more flexible for SUs under the ATP constraint than under the PTP one, and the capacity under the PTP+ATP+PIP+AIP constraints saturates earlier than that under the PTP+PIP and the ATP+PIP constraints because it is restricted by the AIP constraint. Finally, Fig. 4 shows that due to the same reasons as for the results in Fig. 3, the capacity achieved under the ATP+AIP constraints is larger than that 


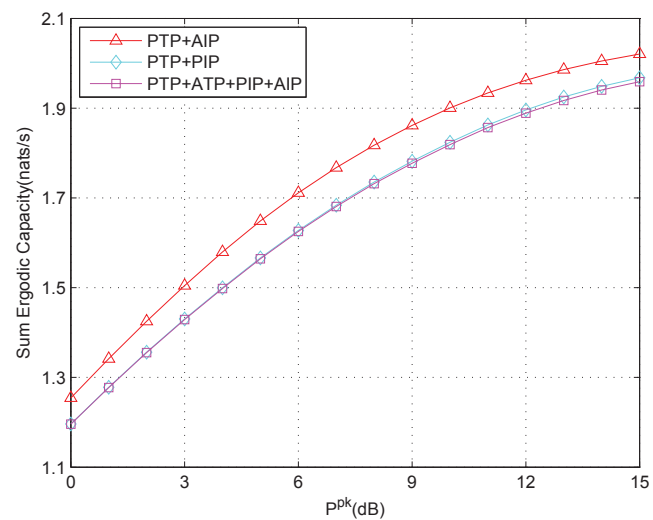

Fig. 1. Sum ergodic capacity vs $P^{p k}$.

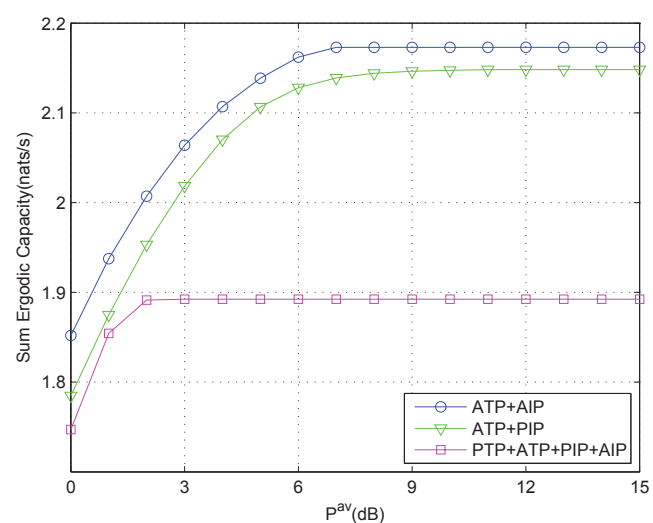

Fig. 2. Sum ergodic capacity vs $P^{a v}$.

achieved under the PTP+AIP constraints and the capacity under the $\mathrm{PTP}+\mathrm{ATP}+\mathrm{PIP}+\mathrm{AIP}$ constraints saturates earlier than that for the PTP+AIP and the ATP+AIP constraints.

\section{CONCLUSION}

The maximum achievable sum ergodic capacity of all SUs in a cognitive radio network, in which multiple SUs share the licensed spectrum of a PU using the FDMA scheme, has been studied subject to the total bandwidth constraint of the licensed spectrum and all possible combinations of the peak/average transmit power constraints at the SUs and interference power constraint imposed by the PU. It has been shown that the bandwidth and power allocation problems can be decoupled. The optimal bandwidth allocation can be found in closed-form given optimal power allocation, while computationally efficient algorithms (see [7]) can be designed for optimal power allocation under different combinations of the power constraints. The structures of the optimal solutions for power allocation have been found.

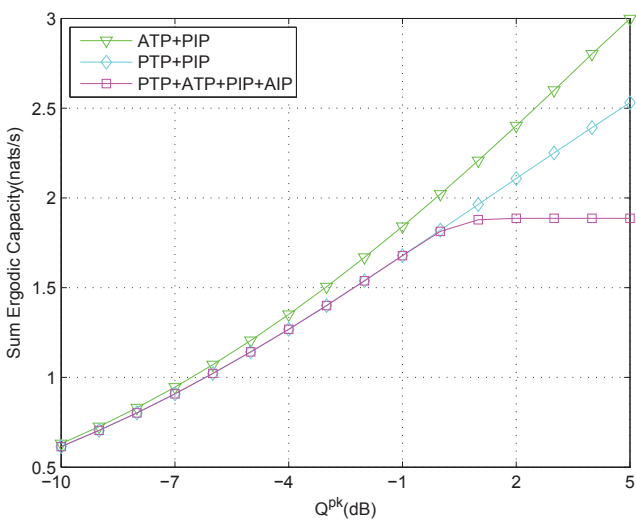

Fig. 3. Sum ergodic capacity vs $Q^{p k}$.

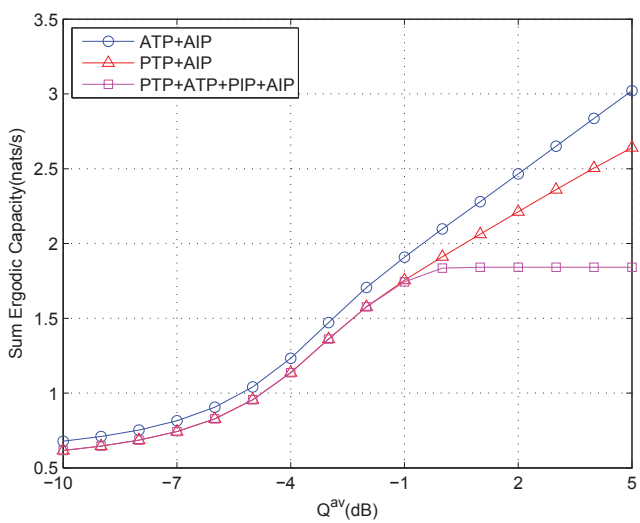

Fig. 4. Sum ergodic capacity vs $Q^{a v}$.

\section{REFERENCES}

[1] Q. Zhao and B. M. Sadler, "A survey of dynamic spectrum access", IEEE Signal Process. Mag., vol. 24, no. 2, pp. 79-89, May 2007.

[2] K. T. Phan, S. A. Vorobyov, N. D. Sidiropoulos, and C. Tellambura, "Spectrum sharing in wireless networks via QoS-aware secondary multicast beamforming," IEEE Trans. Signal Process., vol. 57, no. 6, pp. 2323-2335, Jun. 2009.

[3] L. Musavian and S. Aissa, "Capacity and power allocation for spectrum-sharing communications in fading channels", IEEE Trans. Wireless Commun., vol. 8, no. 1, pp. 148-156, Jan. 2009.

[4] R. Zhang, S. Cui, and Y.-C. Liang, "On ergodic sum capacity of fading cognitive multiple-access and broadcast channels", IEEE Trans. Inform. Theory, vol. 55, no. 11, pp. 5161-5178, Nov. 2009.

[5] E. Biglieri, J. Proakis, and S. Shamai(Shitz), "Fading channels: information-theoretic and communications aspects", IEEE Trans. Inform. Theory, vol. 44, no. 6, pp. 2619-2692, Oct. 1998.

[6] X. Gong, S. A. Vorobyov, and C. Tellambura, "Joint bandwidth and power allocation with admission control in wireless multi-user networks with and without relaying," IEEE Trans. Signal Process, to appear in 2011. (arXiv: CS.IT/1006.1377)

[7] X. Gong, S. A. Vorobyov, and C. Tellambura, "Optimal bandwidth and power allocation for sum ergodic capacity under fading channels in cognitive radio networks," IEEE Trans. Signal Process, to appear in 2011. (arXiv: CS.IT/1006.5061) 